Classification

Physics Abstracts

84.30JE - 84.60JT

\title{
Optimisation du fonctionnement d'un générateur photovoltaïque : Asservissement extrémal de la puissance
}

\author{
C. Boisvineau, M. Nougaret et J. Perard \\ Laboratoire d'Electrotechnique de Grenoble (*), BP 46, 38402 Saint Martin d'Hères, France
}

(Reçu le 20 juillet 1981, révisé le 21 décembre 1981, accepté le 17 février 1982)

\begin{abstract}
Résumé. - La caractéristique statique courant-tension d'un générateur photovoltaïque présente un point de fonctionnement où la puissance électrique débitée dans la charge est maximale. Les coordonnées de ce point dépendent de nombreux paramètres dont l'éclairement, la température, l'état de vieillissement des cellules. Pour fonctionner à tout instant à la puissance maximale, nous adaptons la charge à courant continu $(1,5 \mathrm{~kW})$ à l'aide d'un hacheur à transistor puis nous réalisons sur ce système un asservissement extrémal. Par principe, ce dispositif recherche en permanence le point de fonctionnement optimal quelle que soit l'origine des perturbations qui le modifient.
\end{abstract}

\begin{abstract}
On the static current-voltage characteristic of a photovoltaic generator there is an operating point where the electric power transmitted to the load is maximum. The location of this point depends on many parameters (illumination, temperature of the cells). To obtain the maximum power at each time we first adapt the D.C. load $(1.5 \mathrm{~kW})$ using a transistor chopper. We then control this system with a maximum power point tracking device so that the generator operates at the optimum point whatever the disturbances may be.
\end{abstract}

1. Introduction. - Le coût élevé des générateurs photovoltaïques peut justifier l'adjonction d'un appareillage annexe, même sophistiqué, permettant de gérer au mieux l'énergie disponible. En particulier, sur la caractéristique courant-tension du générateur (qui dépend des conditions d'éclairement, de température, de vieillissement) il existe un point de fonctionnement où la puissance débitée est maximale (Fig. 1). L'optimisation consiste à réaliser ce point en permanence en agissant de façon automatique sur la charge vue par le générateur. Cette adaptation de charge, dont le principe est maintenant classique [1], [2], [3], s'effectue en général à l'aide d'un convertisseur statique dont les pertes doivent être aussi faibles que possible et qui peut, par ailleurs, assurer une fonction de mise en forme d'une grandeur de sortie (conversion continu-alternatif ou continu-continu avec modification de tension par exemple). Différentes attitudes peuvent être envisagées quant à la commande de l'adaptateur. Tout d'abord, la tension $V_{\mathrm{P}}$ du point de puissance maximale dépendant peu de l'éclairement (Fig. 1) une solution simple consiste à asservir la tension du générateur à une valeur de consigne fixe $V_{c}$ (Fig. 2). Un fonctionnement analogue est obtenu sans convertisseur à l'aide de la classique batterie tampon de f.e.m. $E \simeq V_{\mathrm{p}}$. La recherche de l'optimisation peut ensuite être poursuivie en prenant en compte les variations de $V_{\mathrm{P}}$ avec la tempé-

$\left(^{*}\right)$ E.R.A. C.N.R.S. No 534.

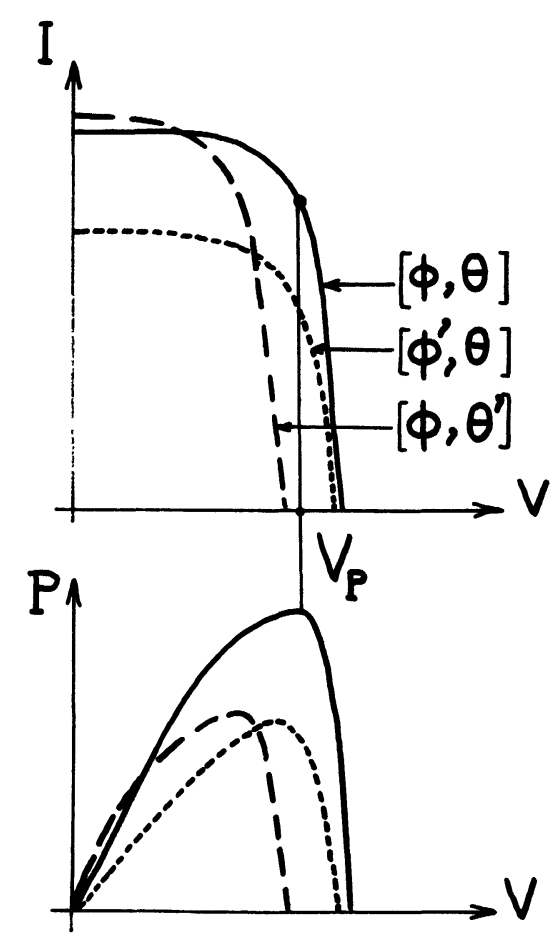

Fig. 1. - Allure des caractéristiques courant-tension et puissance-tension d'un générateur photovoltaïque pour différentes valeurs du flux solaire $\left(\phi, \phi^{\prime}\right)$ et de la température $\left(\theta, \theta^{\prime}\right)$.

[Current-voltage and power-voltage characteristics of a photovoltaic generator with different values of illumination $\left(\phi, \phi^{\prime}\right)$ and temperature $\left(\theta, \theta^{\prime}\right)$.] 


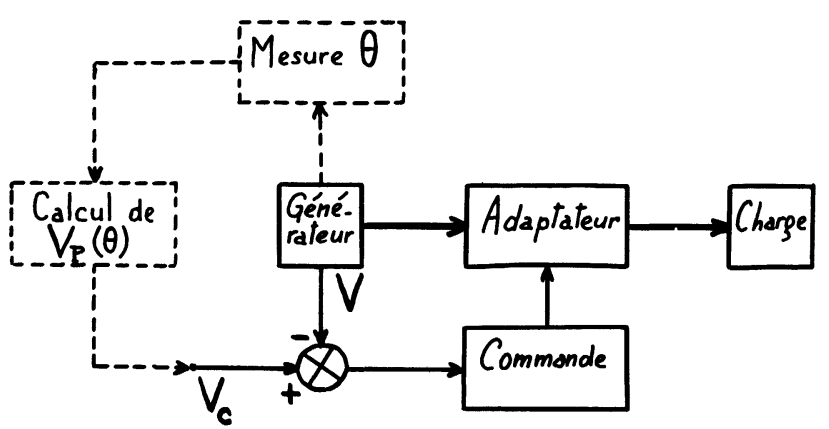

Fig. 2. - Schéma bloc de l'asservissement de tension du générateur d'un système photovoltaĩque et suivi du point de puissance maximale en fonction de la température (partie en pointillés).

[Voltage control block diagram of the photovoltaic generator and maximum power point tracking versus temperature (dashed lines part).]

rature des cellules $\theta$; celle-ci est mesurée en permanence et un modèle permet d'élaborer une consigne égale à $V_{\mathrm{P}}(\theta)$ (méthode d'optimisation avec modèle) (Fig. 2) [1], [4]. Si, plus généralement, on désire suivre le point de puissance maximale en s'affranchissant de la connaissance des paramètres qui modifient sa position, on doit recourir aux techniques d'asservissement extrémal [5]. Cette méthode a déjà été proposée par certains auteurs et, à notre connaissance, a été mise en ouvre, en France, dans le cas d'applications terrestres, sur simulateur [6] et sur un montage de faible puissance [7]. L'intérêt d'un tel dispositif est son universalité vis-à-vis de la nature des perturbations qui affectent la valeur de $V_{\mathrm{P}}$ (par exemple la modification éventuelle des caractéristiques résultant du vieillissement des cellules photovoltaïques sera prise en compte). En effet, le principe de l'asservissement extrémal (Fig. 3) consiste à modifier périodiquement

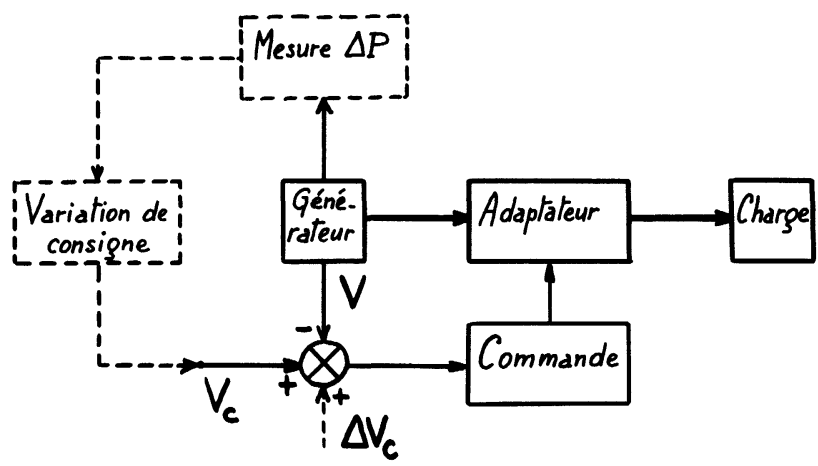

Fig. 3. - Schéma bloc de l'asservissement extrémal de la puissance.

[Maximum power point tracking block diagram.]

la valeur de consigne et à mesurer la variation correspondante de la puissance issue du générateur, afin de déterminer dans quel sens doit évoluer la consigne pour que le fonctionnement se rapproche du point de puissance maximale (problème dit du "MPPT » ou " Maximum Power Point Tracking »). Dans ce cadre, nous proposons de rapporter les principaux éléments d'une réalisation relative à un convertisseur continucontinu adaptateur, à transistor, de $1,5 \mathrm{~kW}$, commandé par asservissement extrémal de la puissance. Cette étude sera complétée par les premiers résultats expérimentaux recueillis sur un système générateur photovoltaïque-adaptateur-motopompe centrifuge.

2. Le convertisseur. - Le convertisseur continucontinu est un hacheur à transistor de type parallèle donc élévateur de tension, dont le schéma de principe est représenté figure 4 . Il est dimensionné pour une

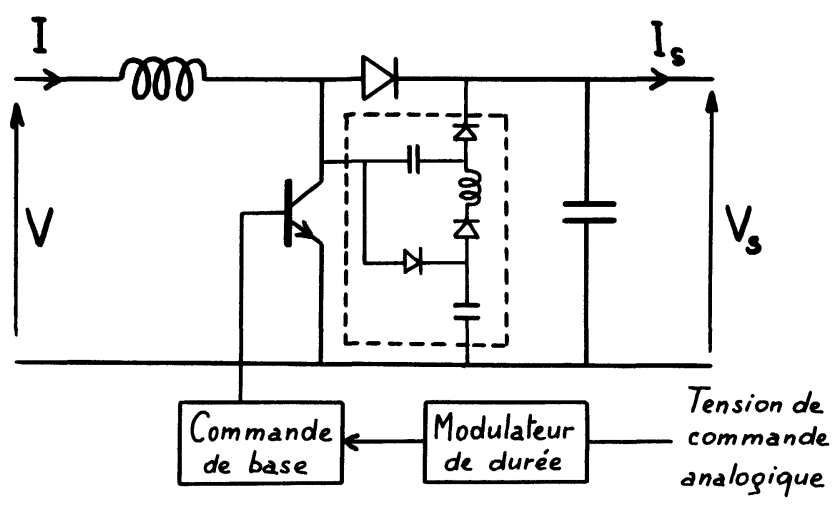

Fig. 4. - Le hacheur à transistor et son circuit d'aide à la commutation.

[The transistor chopper and its switching aid device.]

puissance de $1,5 \mathrm{~kW}$ correspondant aux valeurs nominales $I=30 \mathrm{~A}, V=48 \mathrm{~V}$, côté générateur, $V_{\mathrm{S}}=96 \mathrm{~V}$, $I_{\mathrm{S}}=15 \mathrm{~A}$, côté charge. Son rapport de transformation est, en appelant $r$ le rapport cyclique (rapport du temps pendant lequel le transistor est fermé, à la période de hachage) :

$$
\frac{V_{\mathrm{S}}}{V}=\frac{1}{1-r} .
$$

La valeur de $r$ est élaborée par un modulateur de durée fonctionnant à $15 \mathrm{kHz}$ et commandé par une tension analogique. La commande de base est classique mais munie de tous les circuits assurant la sûreté de fonctionnement du transistor (protection active en courant, inversion de courant base, diode d'antisaturation). Des renseignements détaillés relatifs au convertisseur sont fournis par la référence [8].

Le problème du rendement étant primordial, le circuit de puissance a été conçu de façon à minimiser les pertes de toutes origines en adoptant parfois des solutions ne correspondant pas aux critères habituels de choix des composants qui ont dû être surdimensionnés. Considérons par exemple le cas du transistor de puissance. Un transistor unique a été préféré au Darlington afin de réduire les pertes de conduction. Ce transistor (ESM 1000, $150 \mathrm{~A}, 100 \mathrm{~V}$ ) est lui-même 
utilisé très en dessous de ses possibilités $(30 \mathrm{~A}, 48 \mathrm{~V}$ au lieu de $150 \mathrm{~A}, 100 \mathrm{~V}$ ) de façon à minimiser :

- les pertes de conduction par réduction de la tension de saturation $\left(V_{\text {CE sat }}=0,6 \mathrm{~V}\right.$ à $I_{\mathrm{c}}=30 \mathrm{~A}$ et $I_{\mathrm{b}}=1 \mathrm{~A}$ au lieu de $1,2 \mathrm{~V}$ pour un transistor au courant nominal),

- les pertes dé commande par augmentation du gain en courant $\left(I_{\mathrm{c}} / I_{\mathrm{b}}=30\right.$ au lieu de 10 pour un transistor au courant nominal).

Les pertes de commutation sont réduites par utilisation d'un circuit d'aide à la commutation sans perte [9] représenté sur la figure 4.

Pour les mêmes raisons, la diode est elle-même surdimensionnée et l'inductance est bobinée en fil divisé sur noyau de ferrite.

Dans ces conditions le bilan des pertes s'établit ainsi, pour un fonctionnement à puissance nominale $(1,5 \mathrm{~kW})$ et un rapport cyclique 0,5 :

\section{Transistor :}

Pertes de conduction :

Pertes de commande :

Pertes de commutation

Diode :

Inductance :

Alimentation des circuits électroniques :

Total des pertes :

Le rendement du convertisseur est donc au moins $97 \%$ à puissance nominale. Notons que seules les pertes de commande du transistor et la puissance nécessaire à l'alimentation des circuits électroniques restent constantes lorsque la puissance convertie diminue. Ainsi au dixième de la puissance nominale $(150 \mathrm{~W})$, le rendement peut être encore évalué à $90 \%$.

Enfin, conformément au schéma synoptique général de la figure 3 , le convertisseur est muni d'un asservissement de la tension d'entrée $V$ (tension du générateur) à une valeur de consigne $V_{\mathrm{c}}$. Toute cette partie de l'étude qui a nécessité en particulier la modélisation du convertisseur afin de définir les correcteurs de l'asservissement a déjà été publiée dans la référence [10]. Notons toutefois que cette étude avait été effectuée avec une charge constituée d'une batterie et d'une inductance de lissage et que nous avons conservé le même asservissement dans la réalisation définitive.

3. L'asservissement extrémal. - Le processus sur lequel doit porter l'asservissement extrémal est l'ensemble [générateur-adaptateur-charge] muni de l'asservissement de la tension d'entrée $V$ à la consigne $V_{\mathrm{c}}$. Ces deux tensions $V$ et $V_{\mathrm{c}}$ seront maintenant confondues et notées $V$, ce qui suppose que le temps de réponse du processus à une modification de consigne reste faible devant la période d'échantillonnage mise en jeu par l'asservissement extrémal. La grandeur d'entrée est donc $V$ et la grandeur de sortie $P$, puissance cédée à la charge. La figure 5 représente l'allure de la carac-

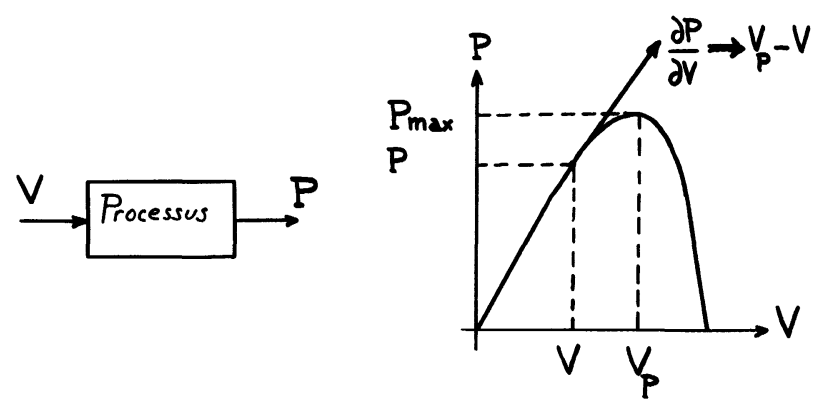

Fig. 5. - Caractéristique puissance-tension du processus et approximation parabolique au voisinage du maximum.

[Power-voltage characteristic of the process and parabolic approximation near the maximum power point.]

téristique statique du processus pour des entrées secondaires données. Remarquons que cette caractéristique est maintenant indépendante de la charge. L'objectif de l'asservissement extrémal est de faire évoluer $V$ vers la valeur $V_{\mathrm{P}}$ correspondant à la puissance maximale $P_{\max }$ et de l'y maintenir en cas de perturbations des entrées secondaires.

3.1 PrINCIPE. - Assimilons la caractéristique statique à une parabole, au voisinage de son sommet (Fig. 5). Nous avons :

$$
P-P_{\max }=a\left(V_{\mathrm{p}}-V\right)^{2} \quad(a<0)
$$

et pour un point de fonctionnement donné du processus, la pente s'écrit :

$$
\frac{\partial P}{\partial V}=-2 a\left(V_{\mathbf{P}}-V\right) .
$$

Ainsi, la valeur de la pente donne une information sur la valeur de l'écart $\left(V_{\mathrm{p}}-V\right)$. L'asservissement extrémal peut donc être considéré d'une manière générale, comme un asservissement à une valeur de consigne où le gradient de la puissance $P$ est utilisé comme signal d'écart, lequel s'annule lorsque l'extrémum est atteint.

3. 2 Structure et FONCTIONNEMENT. - La structure générale de l'asservissement extrémal est représentée figure 6. L'écart est élaboré en effectuant pen-

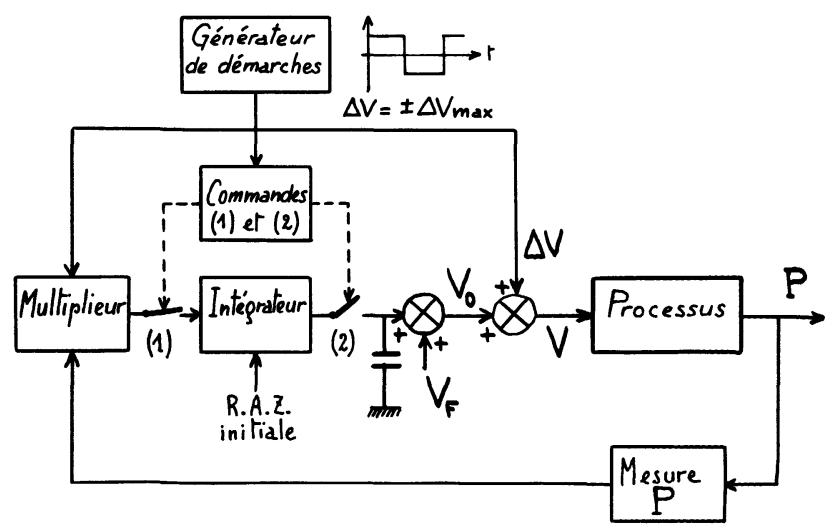

Fig. 6. - Structure du dispositif d'asservissement extrémal.

[Block diagram of the extremum control device.] 
dant un intervalle de temps $\Delta t$ une démarche $\Delta V$, en observant la variation correspondante $\Delta P$ èt en déterminant $\Delta P / \Delta V$ qui permet alors de modifier la consigne.

L'amplitude de la démarche doit être faible pour ne pas perturber le fonctionnement mais une valeur minimale est imposée par la présence des bruits et des perturbations. Ces dernières introduisent en effet une erreur systématique; $P$ étant une fonction de $V$ et des entrées secondaires $p_{i}$, la variation $\Delta P$ observée est :

$$
\Delta P=\frac{\partial P}{\partial V} \Delta V+\sum_{i} \frac{\partial P}{\partial p_{i}} \Delta p_{i}
$$

Cette erreur est d'autant plus faible que le pas de recherche $\Delta V$ est important et que les variations $\Delta p_{i}$, donc l'intervalle $\Delta t$, sont petites. Ce sont les performances dynamiques du processus qui limitent $\Delta t$ vers les faibles valeurs.

Une détection synchrone permet de déterminer l'écart. Ainsi, le signal d'écart est une grandeur échantillonnée égale à la valeur moyenne, sur une période $T$ du signal de recherche, du produit de la puissance $P$ par la fonction modulatrice en créneaux $\Delta V= \pm \Delta V_{\max }$ (Fig. 6). Nous avons, en notant $V_{0}$ la valeur moyenne de $V$ :

$$
\begin{aligned}
P & =P_{\max }+a\left(V_{\mathrm{p}}-V\right)^{2}+P_{\text {bruits }} \\
V & =V_{0}+\Delta V \\
\Delta V & = \pm \Delta V_{\max }
\end{aligned}
$$

et après détection synchrone :

$$
\frac{1}{T} \int_{0}^{T} P . \Delta V . \mathrm{d} t=-2 a\left(\Delta V_{\max }\right)^{2}\left(V_{\mathrm{P}}-V_{0}\right) .
$$

Différentes stratégies peuvent alors être envisagées pour modifier la consigne à l'aide de ce signal d'écart. La plus simple, que nous utilisons, consiste à ajouter l'écart à la consigne à la fin de chaque période d'échan- tillonnage. La valeur de consigne à la $n$-ième période d'échantillonnage est donc :

$$
V_{0 n}=V_{0(n-1)}-2 a\left(\Delta V_{\max }\right)^{2}\left(V_{\mathrm{p}}-V_{0(n-1)}\right) .
$$
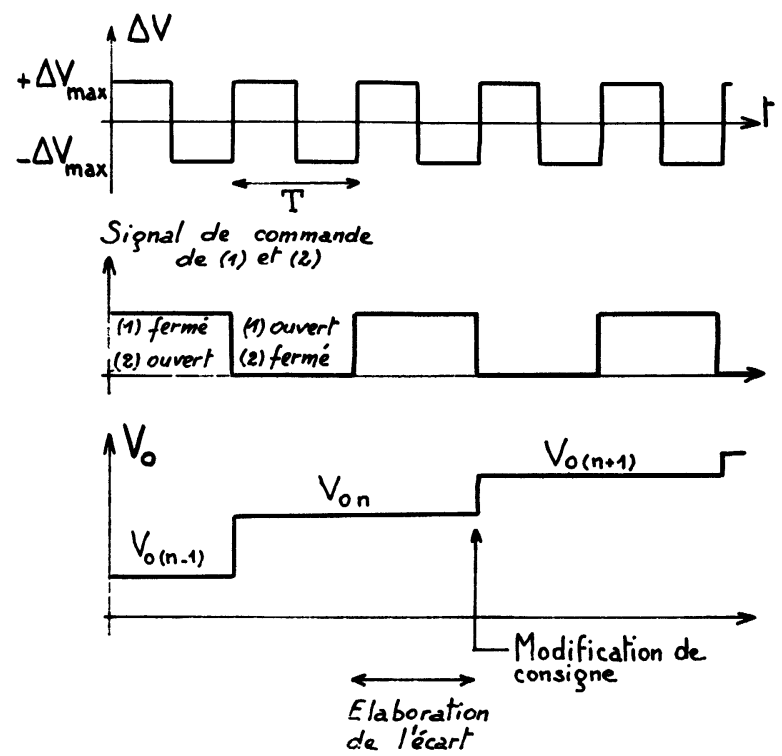

Fig. 7. - Séquence d'élaboration de l'écart et de modification de la consigne.

[Error signal elaboration and control signal modification sequence.]

La figure 7 précise le fonctionnement du dispositif. La recherche de l'extrémum s'effectue à partir d'une valeur initiale fixe $V_{F}$ de la consigne. Celle-ci doit assurer un fonctionnement certain dans les conditions les plus défavorables, en particulier pour la température maximale envisageable qui correspond à la plus faible valeur de $V_{\mathbf{p}}$. La commande complémentaire des interrupteurs (1) et (2) de période $2 T$, est synchronisée sur le signal de recherche. Au cours de deux périodes $T$ successives, le fonctionnement est donc le suivant :

$t \in[k T,(k+1) T[:(1)$ fermé : élaboration de l'écart,

(2) ouvert : la capacité $C$, isolée de la détection synchrone assure le maintien de la consigne.

$t \in[(k+1) T,(k+2) T[:(1)$ ouvert : le multiplieur est isolé de l'intégrateur,

(2) fermé : modification de la consigne.

$V_{0}$ est ainsi inctémenté toutes les deux périodes du signal de recherche et atteint la tension $V_{\mathrm{p}}$ par approches successives si la convergence est assurée.

3.3 CONDITIONS De CONVERGENCE. - Plaçons-nous au voisinage du point de puissance maximale, après $2 k=n$ périodes du signal de démarche, la consigne est :

$$
V_{0 n}=V_{0(n-1)}-2 a \alpha\left(\Delta V_{\max }\right)^{2}\left(V_{p}-V_{0(n-1)}\right)
$$

où le facteur $\alpha$, qui sera explicite par la suite, permet de tenir compte des gains de différents éléments du dispositif. On déduit de cette relation :

$$
\left(V_{p}-V_{0 n}\right)=\left(V_{p}-V_{0(n-1)}\right)\left(1+2 a \alpha\left(\Delta V_{\max }\right)^{2}\right)
$$

soit :

$$
\left(V_{\mathrm{p}}-V_{0 n}\right)=\left(V_{\mathrm{p}}-V_{\mathrm{F}}\right)\left(1+2 a \alpha\left(\Delta V_{\max }\right)^{2}\right)^{n} .
$$


La condition de convergence de $V_{0}$ vers la valeur $V_{\mathrm{p}}$ est par conséquent :

$$
\left|1+2 a \alpha\left(\Delta V_{\max }\right)^{2}\right|<1 \text {. }
$$

La figure 8 donne l'allure de l'évolution du point de fonctionnement dans le plan $[P, V]$ pour chacun des deux cas [5] :

$$
\begin{aligned}
-1 & <1+2 a \alpha\left(\Delta V_{\max }\right)^{2}<0 \\
0 & <1+2 a \alpha\left(\Delta V_{\max }\right)^{2}<1 .
\end{aligned}
$$

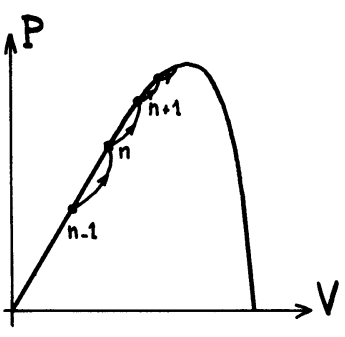

a

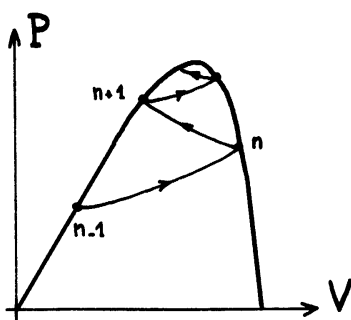

$b$
Fig. 8. - Evolution du point de fonctionnement dans le plan $[P, V]$ dans les deux cas : $a: 0<1+2 a \alpha\left(\Delta V_{\max }\right)^{2}<1$; $b:-1<1+2 a \alpha\left(\Delta V_{\max }\right)^{2}<0$.

[Operating point evolution in the $[P, V]$ diagram in the two cases : $a: 0<1+2 a \alpha\left(\Delta V_{\max }\right)^{2}<1$; $b:-1<1+2 a \alpha\left(\Delta V_{\max }\right)^{2}<0$.]

Nous avons retenu la seconde solution qui évite, lors des perturbations, les oscillations autour de l'extrémum. Le facteur $\alpha(\alpha>0$ pour la solution choisie) est exprimé à partir du schéma bloc de la figure 9 .

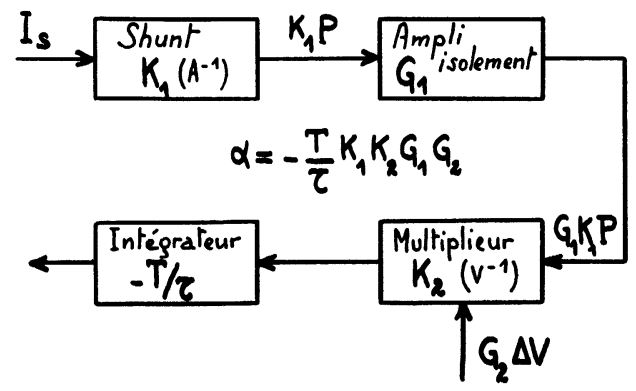

Fig. 9. - Détermination du paramètre $\alpha$.

[Block diagram for $\alpha$ calculation.]

Il est à noter que la mesure de puissance y est remplacée par une mesure du courant de sortie $I_{s}$, c'est donc cette grandeur qui va être rendue maximale. Il en sera en général de même de la puissance car la plupart des récepteurs usuels absorbent une puissance fonction croissante du courant. Cette méthode serait en défaut dans le cas d'un récepteur absorbant un courant constant, il suffirait alors de remplacer la mesure de courant par une mesure de la tension de sortie. Nous avons donc, avec les notations de la figure 9 :

$$
\alpha=-\frac{T}{\tau} K_{1} K_{2} G_{1} G_{2} \quad\left(\text { où } G_{2}<0\right) .
$$

Nous venons d'étudier la convergence au voisinage du point de puissance maximale. Si le point de fonctionnement est éloigné de celui-ci, la loi $P(V)$ peut être approchée par une relation linéaire et il est alors facile de montrer que le point de fonctionnement évolue vers la zone de puissance maximale sans oscillation si la condition de convergence précédemment trouvée est vérifiée. Nous donnons en annexe une étude plus générale de la condition de convergence.

3.4 SCHÉMA ET VALEURS NUMÉRIQUES. - Un schéma simplifié des circuits permettant de réaliser l'asservissement extrémal est donné figure 10. Le signal de recherche est élaboré à partir d'un quartz $1,8 \mathrm{MHz}$. L'ensemble " diviseur de fréquence, bascule » délivre un signal carré de valeur moyenne nulle et de fréquence 2,5 Hz. La commande des interrupteurs analogiques (1) et (2) est synchronisée sur ce signal par l'intermédiaire d'une bascule JK. La détection synchrone utilise un multiplieur intégré et un intégrateur à amplificateur opérationnel. La mesure du courant de sortie, image de la puissance de sortie, est effectuée par un shunt coaxial dont la tension est traitée par un amplificateur d'isolement.

Les valeurs des paramètres permettant de calculer $\alpha$ puis de vérifier la condition de convergence sont :

$$
\begin{aligned}
K_{1}=0,4 \times 10^{-3} \mathrm{~A}^{-1} ; & K_{2} & =0,1 \mathrm{~V}^{-1} \\
G_{1}=70 ; & G_{2} & =-16 \\
T=0,4 \mathrm{~s} ; & \tau & =1,5 \mathrm{~s} \\
\Delta V_{\max }=0,5 \mathrm{~V} ; & 2 a & =-65 \mathrm{~A} \cdot \mathrm{V}^{-1}
\end{aligned}
$$

d'où :

$$
1+2 a \alpha\left(\Delta V_{\max }\right)^{2}=0,82 .
$$

Remarquons que la valeur de $K_{1}$ est mal définie car elle dépend de la tension de sortie. Il en est de même de la valeur de $a$ qui varie avec la caractéristique $P(V)$ à prendre en compte dans des conditions données. (Il suffit de choisir la plus grande valeur.)

4. Résultats expérimentaux. - La mise au point du dispositif et la vérification du fonctionnement (poursuite effective du point de puissance maximale, comportement dynamique) ont été effectuées en laboratoire à l'aide d'un générateur simulé. Celui-ci est constitué d'une source de courant continu stabilisée réglable en parallèle avec un ensemble de diodes lui conférant une caractéristique statique analogue à celle d'un générateur photovoltaïque. (Par contre les capacités des modèles dynamiques sont sensiblement différentes.) La charge est une batterie en parallèle avec 


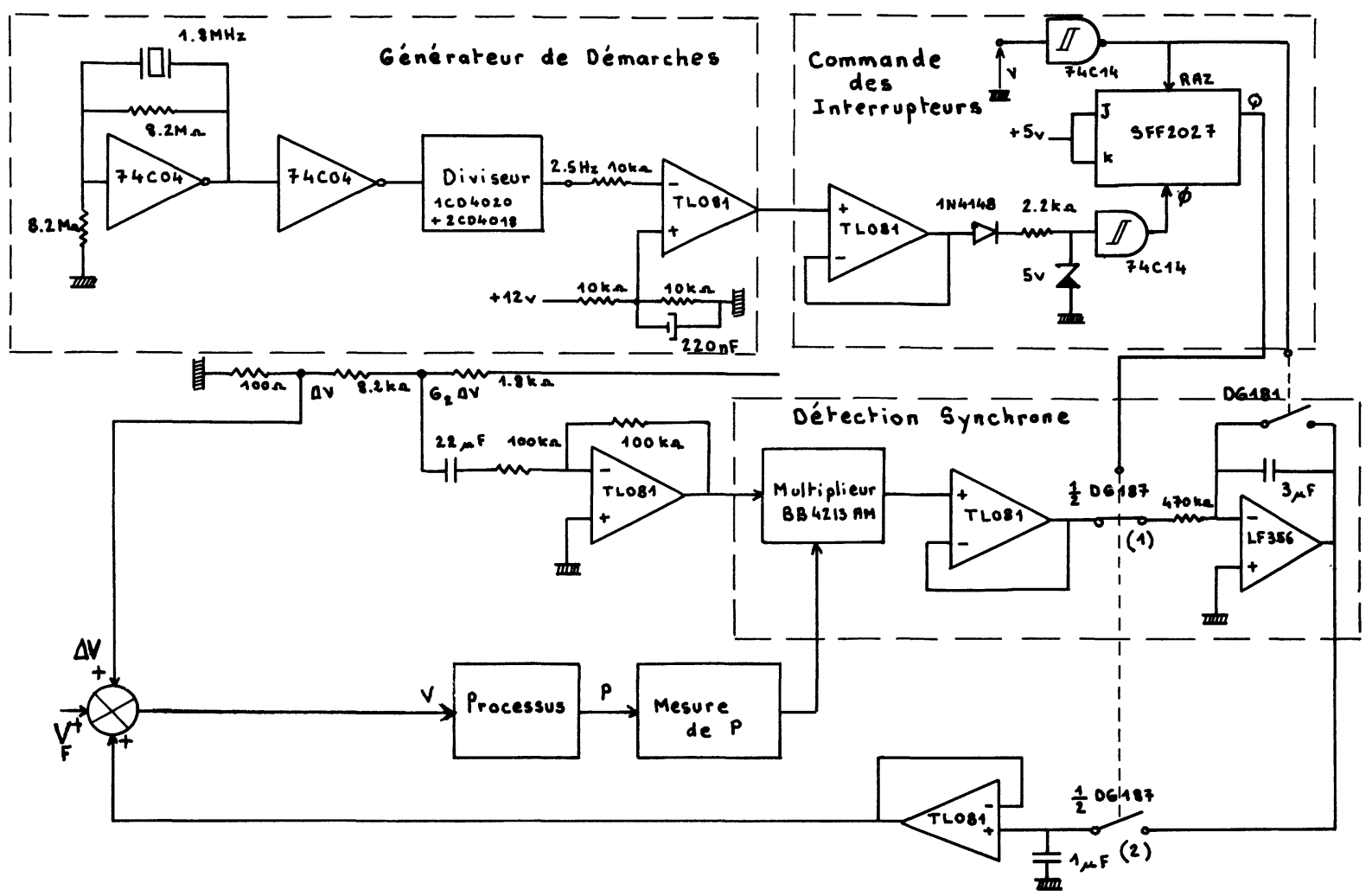

Fig. 10. - Schéma du dispositif d'asservissement extrémal.

[Maximum power point tracking device.]

une résistance. Cette simulation permet de générer facilement des perturbations d'amplitude connue; une modification de la source de courant simule une variation d'éclairement; le court-circuit de quelques diodes simule une variation de température. Des exemples de réponses à ces deux types de perturbations sont fournis par la figure 11 qui représente l'évolution du terme correctif (lequel ajouté à la valeur initiale $V_{\mathrm{F}}$ constitue la consigne) et montre que les temps de réponse mis en jeu sont satisfaisants vis-à-vis de la len-

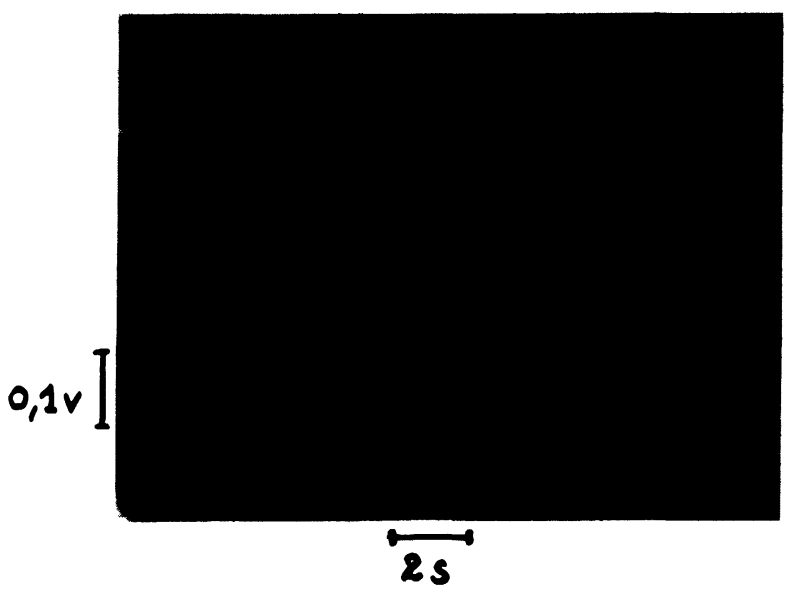

Fig. 11. - Réponse du système aux perturbations : $\left(V_{0}-V_{\mathrm{F}}\right) . a:$ Variation de température (simulée) $; b$ : Variation d'éclairement (simulée). teur des perturbations qui modifieront le fonctionnement du générateur photovoltaïque.

Le convertisseur a été ensuite expérimenté sur un générateur photovoltaïque installé au Centre d'Etudes Nucléaires de Grenoble afin de comparer les fonctionnements d'un système avec et sans adaptateur à asservissement extrémal.

La charge est constituée d'un moteur à aimant permanent $(83 \mathrm{~V}, 14,5 \mathrm{~A}, 3000 \mathrm{tr} / \mathrm{min}$. au régime nominal) entraînant une pompe centrifuge. La position de la

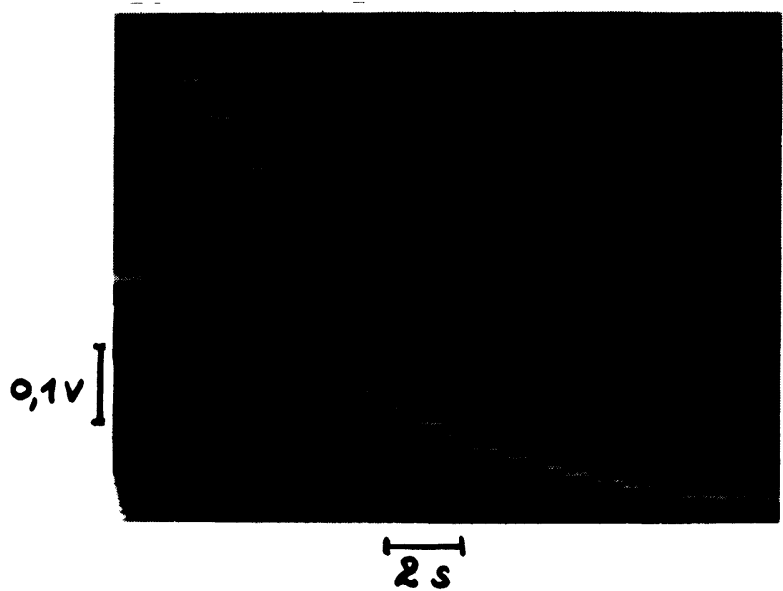

[System response to disturbances. $a$ : Change of temperature (simulation) $b$ : Change of illumination (simulation).] 
caractéristique vue de l'entrée du moteur peut être modifiée par action sur la hauteur manométrique.

Le générateur est constitué de deux panneaux de 28 modules BPX47B18 de RTC. Ces panneaux sont associés en série $\left(15 \mathrm{~A}, 72 \mathrm{~V}\right.$ sous $\left.1 \mathrm{~kW} / \mathrm{m}^{2}\right)$ lors de l'alimentation directe du moteur; en parallèle $(30 \mathrm{~A}$, $36 \mathrm{~V}$ sous $1 \mathrm{~kW} / \mathrm{m}^{2}$ ) lorsque le moteur est alimenté par l'intermédiaire de l'adaptateur qui fonctionnera donc avec un rapport cyclique voisin de 0,5 . La hauteur de pompage a été réglée pour qu'en alimentation directe l'adaptation soit réalisée pour le flux solaire maximal $\left(800 \mathrm{~W} / \mathrm{m}^{2}\right.$ environ pendant la période des essais). La figure 12 donne la puissance à l'entrée du

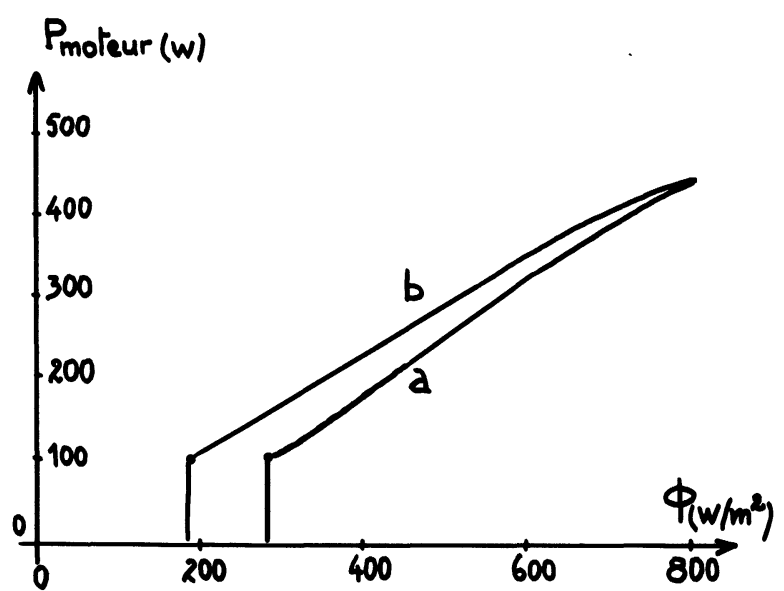

Fig. 12. - Résultats expérimentaux : puissance fournie à une motopompe en fonction du flux solaire. $a$ : Couplage direct du générateur $\left(72 \mathrm{~V}, 15 \mathrm{~A}\right.$ sous $\left.1 \mathrm{~kW} / \mathrm{m}^{2}\right)$ au moteur ; $b$ : Couplage du générateur $\left(36 \mathrm{~V}, 30 \mathrm{~A}\right.$ sous $\left.1 \mathrm{~kW} / \mathrm{m}^{2}\right)$ par l'intermédiaire de l'adaptateur.

[Experimental results : power transmitted to a pumping group as a function of solar flux. $a$ : Direct coupling of the generator $\left(72 \mathrm{~V}, 15 \mathrm{~A}\right.$ under $\left.1 \mathrm{~kW} / \mathrm{m}^{2}\right)$ to the motor; $b$ : Coupling of the generator $\left(36 \mathrm{~V}, 30 \mathrm{~A}\right.$ under $\left.1 \mathrm{~kW} / \mathrm{m}^{2}\right)$ to the motor through the adaptator.]

moteur en fonction du flux solaire avec et sans adaptation et suggère quelques commentaires. Evidemment, par suite des conditions de comparaison, c'est aux faibles flux que l'adaptateur présente le plus d'intérêt. En particulier la charge fonctionne à partir de $195 \mathrm{~W} / \mathrm{m}^{2}$ avec adaptateur au lieu de $280 \mathrm{~W} / \mathrm{m}^{2}$ sans adaptateur. Pour avoir une idée du gain énergétique obtenu, nous nous sommes placés dans le cas d'une variation sinusoïdale du flux solaire, de valeur maximale $800 \mathrm{~W} / \mathrm{m}^{2}$ et avons ainsi évalué à $15 \%$ le supplément d'énergie fourni grâce à l'adaptateur. Ce résultat paraît d'autant plus intéressant que la charge est une pompe centrifuge dont la caractéristique intrinsèque est déjà relativement bien adaptée.

5. Conclusion. - Les principes que nous venons de développer s'appliquent à d'autres problèmes du domaine des énergies renouvelables. Un cas semblable est celui d'une chaîne "générateur photovoltaïque- onduleur-machine asynchrone-charge mécanique » [2]; l'adaptation de la charge vue par le générateur doit alors être obtenue par action sur les paramètres de fonctionnement de l'onduleur (fréquence et tension efficace, pour un onduleur à modulation de largeur d'impulsions) en évitant l'emploi de deux convertisseurs en cascade. Citons enfin les générateurs à éoliennes qui présentent aussi une caractéristique de sortie dont la puissance est maximale pour une certaine valeur de la charge et dont le fonctionnement devrait pouvoir être optimisé par asservissement extrémal. On peut toutefois reprocher à ces dispositifs une certaine complexité de l'électronique de commande qui n'est pas en faveur de leur fiabilité. Cet inconvénient devrait rapidement disparaître, en particulier avec l'apparition imminente de circuits intégrant une commande de base complète de transistor de puissance. Outre le convertisseur, le circuit ne comprendrait alors que quelques composants : la commande de base, un microprocesseur réalisant l'asservissement extrémal [7], une alimentation à découpage élaborant les tensions d'alimentation.

6. Annexes. - La condition de convergence peut être définie de façon plus générale. En effet, si l'amplitude du signal de recherche est assez faible pour supposer que la pente de $P(V)$ ne varie pas au cours d'une démarche, la sortie de la détection synchrone fournit à la fin de la $n$-ième période d'échantillonnage :

$$
\left(\frac{\partial P}{\partial V}\right)_{n-1} \cdot \alpha \cdot\left(\Delta V_{\max }\right)^{2}
$$

d'où :

$$
V_{0 n}=V_{0 n-1}+\left(\frac{\partial P}{\partial V}\right)_{n-1} \alpha\left(\Delta V_{\max }\right)^{2} .
$$

Pour avoir convergence vers la valeur $V_{\mathrm{P}}$, sans oscillation de part et d'autre de $V_{\mathrm{P}}$, il faut donc vérifier les conditions suivantes :

$\mathrm{Si}$

$$
\frac{\partial P}{\partial V}>0: \quad V_{0 n-1}<V_{0 n}<V_{p}
$$

soit :

$$
0<\left(\frac{\partial P}{\partial V}\right)_{n-1} \alpha\left(\Delta V_{\max }\right)^{2}<V_{\mathrm{p}}-V_{0 n-1} .
$$

$\mathrm{Si}$

$$
\frac{\partial P}{\partial V}>0: \quad V_{\mathrm{p}}<V_{0 n}<V_{0 n-1}
$$

soit :

$$
V_{\mathrm{p}}-V_{0 n-1}<\left(\frac{\partial P}{\partial V}\right)_{n-1} \alpha\left(\Delta V_{\max }\right)^{2}<0
$$




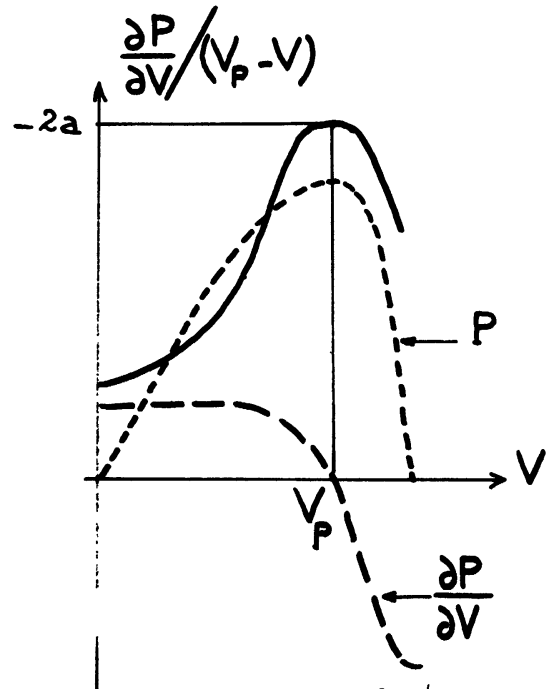

Fig. 13. - Allure des variations de $\frac{\partial P}{\partial V} /\left(V_{\mathrm{p}}-V\right)$ en fonction de $V$ montrant qu'il suffit de vérifier la condition de convergence au voisinage du maximum.

[The representation of $\frac{\partial P}{\partial V} /\left(V_{p}-V\right)$ as a function of $V$ shows that it is sufficient for the convergence condition to be verified near the maximum power point.] donc, sous forme condensée :

$0<\frac{\frac{\partial P}{\partial V}}{V_{\mathrm{p}}-V} \alpha\left(\Delta V_{\max }\right)^{2}<1$.

La figure 13 représente l'allure des variations de $P$, $\frac{\partial P}{\partial V}, \frac{\partial P}{\partial V} /\left(V_{\mathrm{p}}-V\right)$ en fonction de $V$ dans le cas du problème posé et montre qu'il suffit de vérifier la condition au voisinage du maximum où

$$
\frac{\partial P}{\partial V} /\left(V_{\mathrm{p}}-V\right)=-2 a \text {. }
$$

Remerciements. - Les auteurs expriment leurs remerciements à l'équipe de feu Mr Jourdan Paul, du Laboratoire d'Etude des Matériaux Minces (Centre d'Etudes Nucléaires de Grenoble) pour sa collaboration en ce qui concerne la validation du dispositif sur générateur photovoltaïque.

\section{Bibliographie}

[1] Roger, J. A., Pivot, J., "Les générateurs photovoltaïques autonomes sans batteries ". Revue Phys. Appl. 15 (1980) 603-609.

[2] Barlaud, M., Sow, G., Masselot, C., « Alimentation d'une pompe immergée à partir d'un générateur photovoltaïque à l'aide d'un onduleur triphasé à transistors ». Revue Phys. Appl. 15 (1980) 263-269.

[3] Josse, M., Prido, R., "Projet de chaîne de conversion à haut rendement, puissance de 1 à $10 \mathrm{~kW}$ ». Colloque sur les systèmes de conversion photovoltaïques, 4-5 décembre 1978, Toulouse.

[4] Annabi, M., Ben Slama, N., Kachouk, M., Ksouri, M., PeraRD, J., « Identification d'une installation de pompage solaire ". Revue Internationale d'Héliotechnique, 2e semestre 1980.

[5] Decaulne, P., Gille, J. C., Pelegrin, M., « Introduction aux asservissements extrémaux et adaptatifs ». Livre (Dunod Ed.).
[6] Bei, M., Jalade, J., Marpinard, J. C., " Conditionnement de puissance : simulation sur calculateur hybride ". Colloque sur les systèmes de conversion photovoltaïques, 4-5 décembre 1978, Toulouse.

[7] Barlaud, M., Pradal, D., ReQuier, J. P., « Recherche de la puissance optimale délivrée par un générateur photovoltaïque à l'aide d'un dispositif microprogrammé ". Entropie no 95, 1980.

[8] Boisvineau, C., "Optimisation du fonctionnement d'un générateur photovoltaïque ». Thèse ENSIEG, Grenoble, mars 1981.

[9] BOEHRINGER, A., KNOLL, H., "Transistorshalter im Bereich hoher Leistungen und Frequenzen ». Etz Bd 100, Heft 13, pp. 664-669.

[10] Boisvineau, C., Toutain, E., Perard, J., * Modélisation des hacheurs ". Mesures, Régulation, Automatisme (avril 1980), pp. 95-100. 